\title{
Modélisation mécanique et numérique de la mise en forme de structures souples avec remaillage adaptatif
}

\author{
Ilyes Ben Naceur $^{1,2}$, Abdelhakim Cherouat ${ }^{1, a}$, Houman Borouchaki $^{1}$ \\ ET JEAN-MARIE BACHMANN ${ }^{2}$ \\ 1 Laboratoire des systèmes mécaniques et d'ingénierie simultanée, BP 2060, 12 rue Marie Curie, 10010 Troyes, France \\ 2 Institut français de textile et de l'habillement, IFTH, 270 rue du faubourg Croncels, 10042 Troyes Cedex, France
}

Reçu le 25 février 2005, accepté le 21 mars 2005

\begin{abstract}
Résumé - La modélisation de la mise en forme des structures souples présente un outil précieux d'aide à la conception. Elle permet d'étudier la faisabilité du procédé de fabrication ainsi que la prédiction des défauts qui puissent être générés. Cependant, il est nécessaire d'avoir une connaissance du comportement mécanique de la structure. Une modélisation du comportement mécanique des étoffes souples est présentée. L'identification des paramètres du modèle s'appuie sur des essais de traction uni-axiale. Une méthode d'identification par approche inverse basée sur des algorithmes d'optimisation est proposée. Des simulations de mise en forme par emboutissage avec remaillage adaptatif sont étudiées dans cet article.
\end{abstract}

Mots clés : Modèle macroscopique / méthode inverse / remaillage adaptatif / mise en forme

\begin{abstract}
The simulation of the forming of flexible structures presents an invaluable tool of assistance at the design. It makes it possible to study the feasibility of the manufacturing process as well as the prediction of the defects which can be generated. However, it is necessary to study the mechanical behaviour of the structure. A modelling of the mechanical behaviour of the flexible fabrics is presented. The identification of the parameters of the model is based on uni-axial tensile tests. A method of identification by inverse approach based on an optimization algorithm is proposed. Simulations of the deep-drawing of fabrics using adaptive remeshing procedure are also given.
\end{abstract}

Key words: Macroscopic model / inverse approach / adaptive remeshing / forming process

\section{Introduction}

La modélisation du comportement des structures souples destinées à la mise en forme est devenue l'un des objectifs prioritaires de l'industrie textile et composite qui vise à améliorer la productivité et la qualité des produits fabriqués. La modélisation du comportement mécanique des structures tissées et tricotées permet de prédire leur capacité de déformation. De même, la simulation permet de tester la faisabilité des procédés de fabrication ainsi que leur capacité à épouser la forme géométrique souhaitée lors de la mise en forme. Plusieurs approches ont été proposées pour la modélisation du comportement des étoffes. On trouve les approches géométriques qui caractérisent la géométrie et la forme des mailles par des splines en considérant juste les dimensions sans tenir compte des propriétés physiques de l'étoffe [1]. D'autres approches tiennent compte du comportement mécanique des fibres

\footnotetext{
a Auteur correspondant : cherouat@utt.fr
}

et proposent des modèles plus riches qui tiennent compte des propriétés mécaniques [2-4].

$\mathrm{Au}$ niveau des modèles mécaniques, on distingue les approches microscopiques à l'échelle de la fibre [5], les approches mésoscopiques à l'échelle de la maille élémentaire de l'étoffe et les approches macroscopiques à l'échelle de la structure [6]. Dans ce travail, on présente un modèle de comportement à l'échelle macroscopique. Pour caractériser la structure hétérogène de l'étoffe, on propose une discrétisation géométrique par éléments de barre, représentant les fibres, mixés avec des éléments de membrane représentant l'élasthanne. Le comportement mécanique caractérisé par un modèle macroscopique est implémenté dans la plate-forme du code ABAQUS. Les paramètres du modèle sont identifiés par des essais de traction uni-axiale avec par méthode inverse basée sur un algorithme d'optimisation. Au niveau de la simulation, nous proposons une procédure de remaillage adaptatif couplée avec le code de calcul par éléments finis ABAQUS. 
Des simulations de mise en forme par emboutissage sont proposées.

\section{Caractérisation et modélisation du comportement}

Les structures souples tricotées et tissées se distinguent souvent par une grande discontinuité de la matière et aussi par son hétérogénéité à cause de sa composition par plusieurs types de matériau. Pour modéliser le comportement mécanique d'une telle structure nous nous sommes basés sur une approche macroscopique à l'échelle de la structure globale. L'avantage de cette approche, c'est qu'elle puisse être adaptée à des structures avec des liages différents (taffetas, jersey, ...) et avec des matériaux différents. Le modèle de comportement est établi sur la base d'un essai de traction uni-axiale. À partir de la courbe force-déplacement, on déduit la contrainte réelle uni-axiale $\sigma_{\mathrm{L}}$ et la déformation $\varepsilon_{\mathrm{L}}$ par :

$$
\sigma_{\mathrm{L}}=\left(1+\varepsilon_{\mathrm{L}}\right) \frac{F}{S_{0}} \quad \text { et } \quad \varepsilon_{\mathrm{L}}=\frac{\Delta l}{l_{0}}
$$

avec $S_{0}$ est la section initiale de l'éprouvette, $l_{0}$ sa longueur initiale et $\Delta l$ l'allongement de l'éprouvette.

Un essai de traction est réalisé sur une éprouvette en étoffe de dimension $100 \mathrm{~mm} \times 50 \mathrm{~mm}$ avec un dynamomètre supportant jusqu'à $500 \mathrm{kN}$ et une vitesse de chargement égale à $4 \mathrm{~mm} . \mathrm{s}^{-1}$. Cette étoffe est composée de $70 \%$ de polyamide et $30 \%$ d'élasthanne. La courbe de la réponse locale (contrainte-déformation) peut être caractérisée par un polynôme qui dépend de l'allure de la réponse. Pour l'exemple étudié, le modèle de comportement uni-axial est caractérisé par $\sigma_{\mathrm{L}}=f\left(\varepsilon_{\mathrm{L}}\right)$, avec $f$ une fonction non-linéaire de degré 5 représenté par l'équation suivante

$$
\sigma_{\mathrm{L}}=E_{0}+E_{1} \cdot \varepsilon_{\mathrm{L}}+E_{2} \cdot \varepsilon_{\mathrm{L}}^{2}+E_{3} \cdot \varepsilon_{\mathrm{L}}^{3}+E_{4} \cdot \varepsilon_{\mathrm{L}}^{4}+E_{5} \cdot \varepsilon_{\mathrm{L}}^{5}
$$

où $E_{i(i=0,5)}$ sont les paramètres caractéristiques de l'étoffe et sont déterminés à partir des essais de traction.

Les valeurs de ces paramètres sont calculées dans un premier temps par la méthode d'identification au sens des moindres carrés. En effet, on calcule les valeurs des paramètres qui permettent de minimiser le mieux possible les distances entre les points expérimentaux et la courbe numérique. À partir des $N$ points expérimentaux dont on dispose, on résout le système d'équations suivant pour trouver les valeurs des paramètres du modèle :

$$
\left[\begin{array}{cccccc}
1 & \varepsilon_{1} & \varepsilon_{1}^{2} & \varepsilon_{1}^{3} & \varepsilon_{1}^{4} & \varepsilon_{1}^{5} \\
1 & \varepsilon_{2} & \varepsilon_{2}^{2} & \varepsilon_{2}^{3} & \varepsilon_{2}^{4} & \varepsilon_{2}^{5} \\
\cdot & \cdot & \cdot & \cdot & \cdot & \cdot \\
\cdot & \cdot & \cdot & \cdot & \cdot & \cdot \\
\cdot & \cdot & \cdot & \cdot & \cdot & \cdot \\
1 & \varepsilon_{N} & \varepsilon_{N}^{2} & \varepsilon_{N}^{3} & \varepsilon_{N}^{4} & \varepsilon_{N}^{5}
\end{array}\right] \cdot\left(\begin{array}{c}
E_{0} \\
E_{1} \\
E_{2} \\
E_{3} \\
E_{4} \\
E_{5}
\end{array}\right)=\left(\sigma_{i}\right) \quad i=1, \ldots N
$$

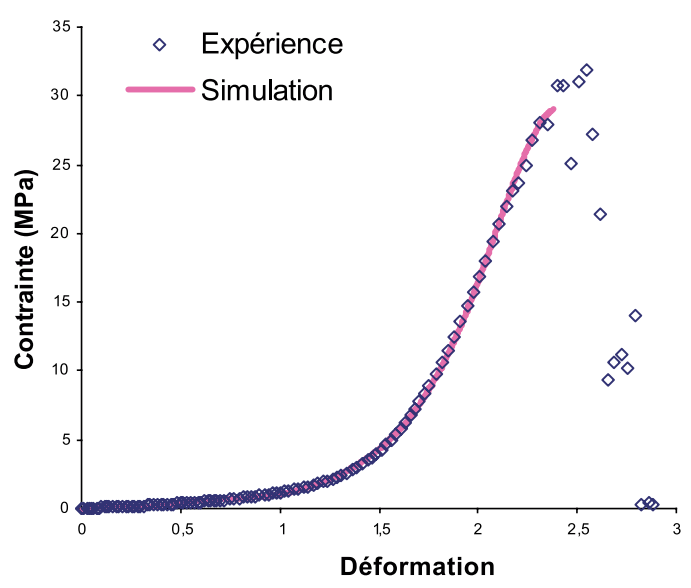

Fig. 1. Réponse locale contrainte-déformation.

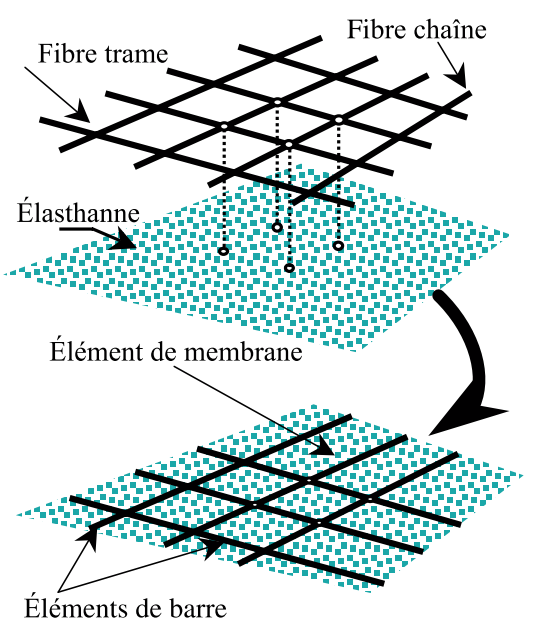

Fig. 2. Discrétisation géométrique d'une maille élémentaire.

Ce modèle de comportement est intégré sur la plateforme d'ABAQUS via une procédure pour caractériser le matériau (UMAT), où il est nécessaire de calculer le module tangent à chaque incrément par :

$$
E_{\mathrm{T}}(\varepsilon)=\frac{\mathrm{d} \sigma_{\mathrm{L}}}{\mathrm{d} \varepsilon_{\mathrm{L}}}
$$

La figure 1 illustre la courbe d'identification en un point matériel en comparaison avec la courbe expérimentale. On remarque bien que la réponse numérique est cohérente avec la réponse expérimentale.

Pour la validation du modèle global au niveau de la structure, une simulation numérique d'un essai de traction uni-axiale est proposée. L'éprouvette en taffetas tissée de dimensions $100 \times 50 \mathrm{~mm}$ est discrétisée par des éléments de barres qui caractérisent les fibres de polyamide et par des éléments de membranes qui caractérisent l'élasthanne (voir Fig. 2). Le modèle de comportement établi $\sigma_{\mathrm{L}}=f\left(\varepsilon_{\mathrm{L}}\right)$ est assigné aux éléments de barres alors que le comportement des éléments de membrane est supposé élastique isotrope linéaire avec un module de Young de $20 \mathrm{MPa}$ et un coefficient de Poisson égal à 0,4 .

Du fait de la discontinuité de la structure et la non-homogénéité de la déformation, la réponse globale 


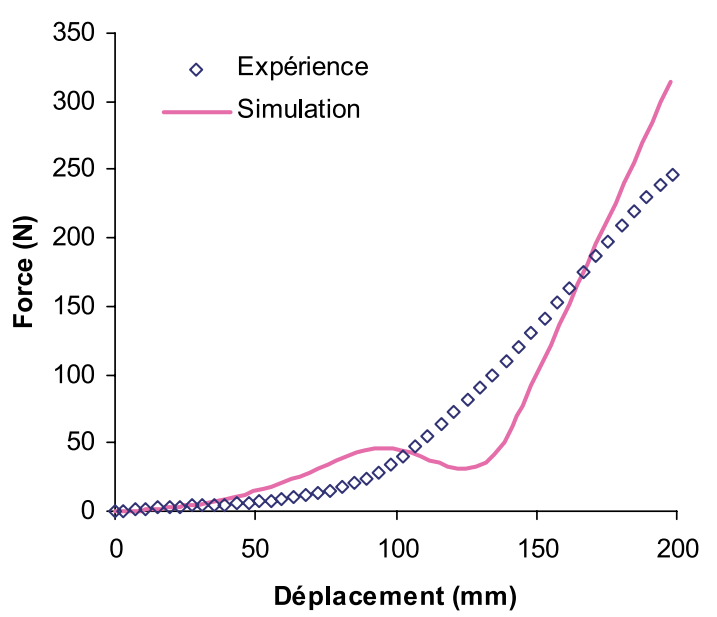

Fig. 3. Réponse globale force-déplacement.

obtenue par simulation de l'essai de traction n'est pas identique à l'expérience (voir Fig. 3). Pour atténuer cette différence, il est nécessaire de déterminer les valeurs des paramètres du modèle qui tiennent compte du comportement local et aussi de l'effet de la structure. Pour cela, une méthode d'identification par approche inverse est proposée. Cette méthode consiste à coupler le code de calcul par éléments finis ABAQUS avec une procédure d'optimisation par algorithme génétique [7]. La minimisation de l'écart entre la courbe expérimentale (force-déplacement) et la courbe numérique représente la fonction objectif de ce problème d'optimisation alors que les contraintes du problème sont les intervalles de variation des paramètres du modèle [8]. Un deuxième code d'optimisation basé sur la méthode Levenberg-Marquardt est utilisé pour l'identification des paramètres matériau, cette méthode est intégrée dans le code ZEBULON [9].

\section{Identification par approche inverse}

L'identification par approche inverse est basée sur un algorithme d'optimisation. Pour cela il faut se fixer une fonction objectif qui permet de minimiser les distances entre les points des deux courbes obtenues expérimentalement et numériquement, les contraintes de ce problème d'optimisation étant les intervalles de variation des paramètres du modèle.

Ce problème d'optimisation sous contrainte est établi en fonction des variables $\left(E_{0}, E_{1}, E_{2}, E_{3}, E_{4}, E_{5}\right)$. La minimisation de l'écart entre les courbes analytique et expérimentale est assurée par la fonction objectif qui s'écrit selon la formulation suivante :

$$
\begin{aligned}
& \operatorname{Minimiser}_{\left(E_{0}, E_{1}, E_{2}, E_{3}, E_{4}, E_{5}\right)}\{\text { erreur }= \\
& \left.\int_{0}^{x_{\max }}\left(F_{\operatorname{sim}}-F_{\exp }\right)^{2} \mathrm{~d} x\right\}
\end{aligned}
$$

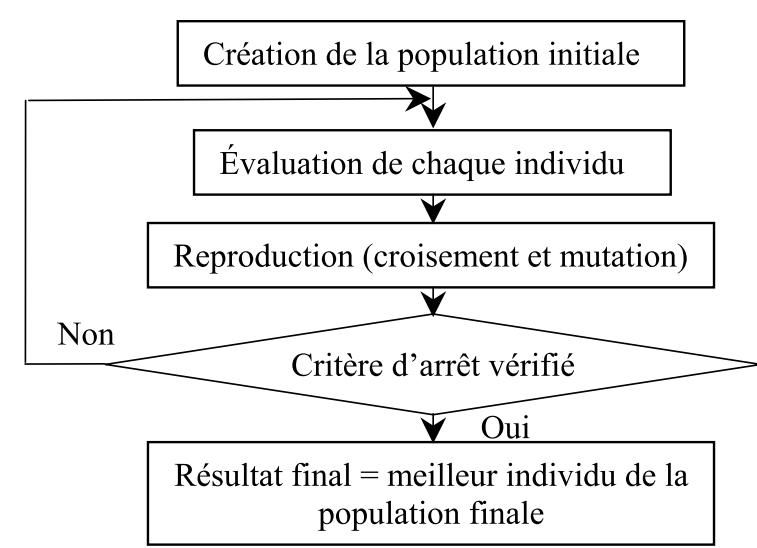

Fig. 4. Organigramme de l'algorithme génétique.

où $F_{\text {sim }}$ est la force calculée sur le bord de l'éprouvette après la simulation et $F_{\text {exp }}$ est la force expérimentale mesurée lors de l'essai de traction. Le paramètre $x$ correspond au déplacement enregistré au cours de l'essai alors que $x_{\max }$ correspond au déplacement maximal. L'erreur est calculée par la méthode des trapèzes. En effet, une interpolation linéaire permet de trouver pour chaque déplacement enregistré la valeur correspondante sur la courbe expérimentale. Ceci permet de calculer facilement l'intégrale de $\left(F_{\text {sim }}-F_{\text {exp }}\right)^{2}$ par la méthode des trapèzes.

Les contraintes sont définies par les intervalles de variation des variables tels que :

$$
E_{i, \min } \leq E_{i} \leq E_{i, \max } \quad i \in[0, \ldots, 5]
$$

Pour résoudre ce problème d'optimisation, deux approches sont appliquées : une approche globale assurée par l'algorithme génétique qui est basé sur les méthodes stochastiques et une approche locale assurée par la méthode de Levenberg-Marquardt.

L'algorithme génétique est basé sur l'évolution vers l'optimum d'une population de paramètres par générations successives. Chaque génération est caractérisée par un nombre d'enfants et de parents, par une probabilité de mutation et de croissement. Un grand nombre de générations permet d'affiner le résultat et un grand nombre de parents et d'enfants permet d'élargir le domaine de recherche de l'optimum [7]. L'organigramme de cet algorithme est présenté sur la figure 4. Chaque population est composée d'un nombre d'individus qui représentent les valeurs des paramètres à optimiser. Ces paramètres sont de type continu et ils sont codés en binaire. Le critère d'arrêt du calcul d'optimisation est défini par le nombre d'exécutions. Une fois le nombre d'exécutions atteint, le calcul s'arrête même si on n'atteint pas une valeur d'erreur minimale. Le jeu de variable optimale est obtenu automatiquement à la fin de l'exécution du code. Afin de calculer pour chaque jeu de variables l'erreur entre expérience et simulation, un couplage est réalisé entre le solveur de calcul par éléments finis (ABAQUS) et l'algorithme génétique selon l'organigramme présenté sur la figure 5 .

La deuxième méthode qui est celle de LevenbergMarquardt est intégrée dans le code ZEBULON [9]. 


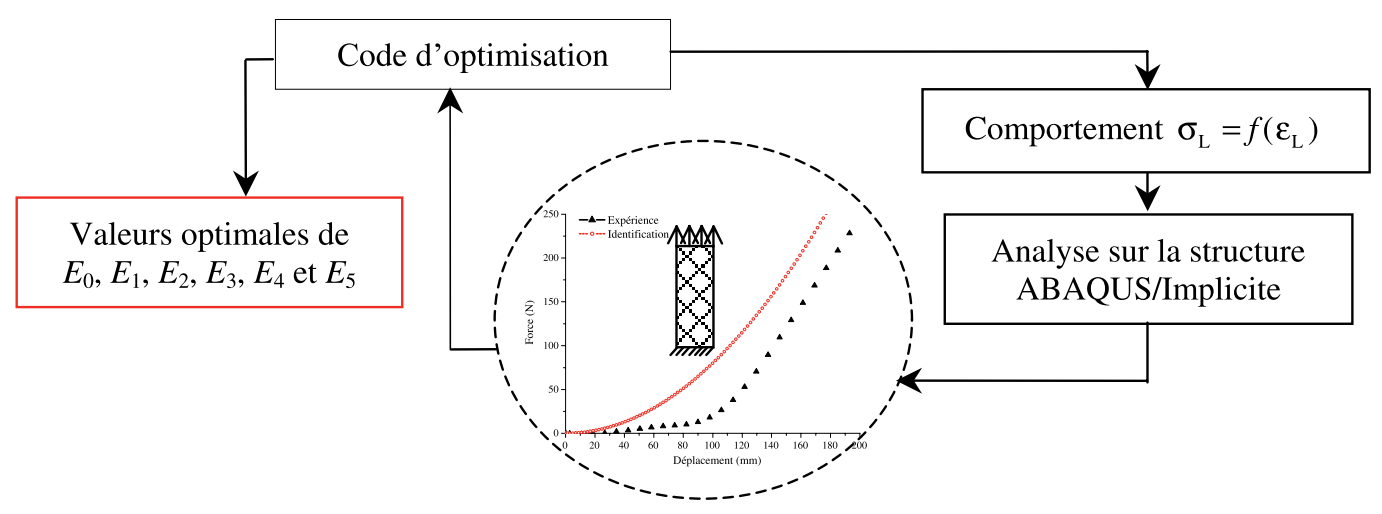

Fig. 5. Organigramme d'identification par méthode inverse.

Tableau 1. Valeurs de l'erreur et des paramètres du modèle au cours des itérations.

\begin{tabular}{llllllll}
\hline Itération $\mathrm{N}^{\circ}$ & Erreur $\left(\mathrm{N}^{2} . \mathrm{mm}\right)$ & $E_{0}$ & $E_{1}$ & $E_{2}$ & $E_{3}$ & $E_{4}$ & $E_{5}$ \\
\hline 1 & 3280 & $-0,119$ & 3,15 & $-6,46$ & 1,01 & 10,1 & $-3,35$ \\
6 & 3350 & $-0,119$ & 3,15 & $-6,46$ & 1,01 & 10,1 & $-3,35$ \\
10 & 708 & $-0,119$ & $-13,0$ & $-17,7$ & 1,20 & 21,9 & $-7,93$ \\
12 & 15,7 & $-0,119$ & $-13,0$ & $-17,7$ & 1,20 & 21,9 & $-7,93$ \\
\hline
\end{tabular}

Cette méthode dite locale est basée sur un calcul approximatif du gradient et du Hessien de la fonction objectif. Cette fonctionnelle est décrite par l'équation suivante :

$$
F(x)=\frac{1}{2} \sum_{i=1}^{N} w_{i}\left(F_{\operatorname{sim}, i}(x)-F_{\exp , i}\right)^{2}
$$

$F_{\text {sim }}$ et $F_{\text {exp }}$ sont respectivement la force numérique et expérimentale et $w_{i}$ sont les poids associés.

Pour la résolution du problème, une simulation d'un essai de traction est réalisée avec le code ABAQUS couplé avec le module d'optimisation de ZEBULON suivant l'organigramme de la figure 5. L'application de ces deux méthodologies d'identification ont abouti quasiment au même résultat (voir Fig. 7) mais avec une large différence au niveau du temps de calcul (45 min pour la méthode de Levenberg-Marquardt et environ $3 \mathrm{~h}$ pour l'algorithme génétique).

En effet, l'algorithme génétique est assez lent pour converger vers les optimums ce qui constitue un inconvénient de cette méthodologie. En revanche cet algorithme garantit une convergence vers l'optimum global ce qui n'est pas le cas de la méthode de LevenbergMarquardt. Le tableau 1 représente l'évolution des erreurs au cours des itérations ainsi que les valeurs des paramètres matériau pour la méthode de Levenberg-Marquardt.

La figure 6 présente l'évolution de la courbe de simulation au cours des itérations, elle tend vers la courbe expérimentale au bout de la $12^{\text {e }}$ itération. Malgré que la méthode de Levenberg-Marquardt constitue un avantage au niveau de la rapidité de convergence elle constitue de même un inconvénient qui est la convergence vers les minimums locaux. L'obtention d'un minimum global n'est pas donc garantie. Pour avoir un résultat rigoureux, il sera nécessaire d'adopter la méthode d'optimisation par algorithme génétique.

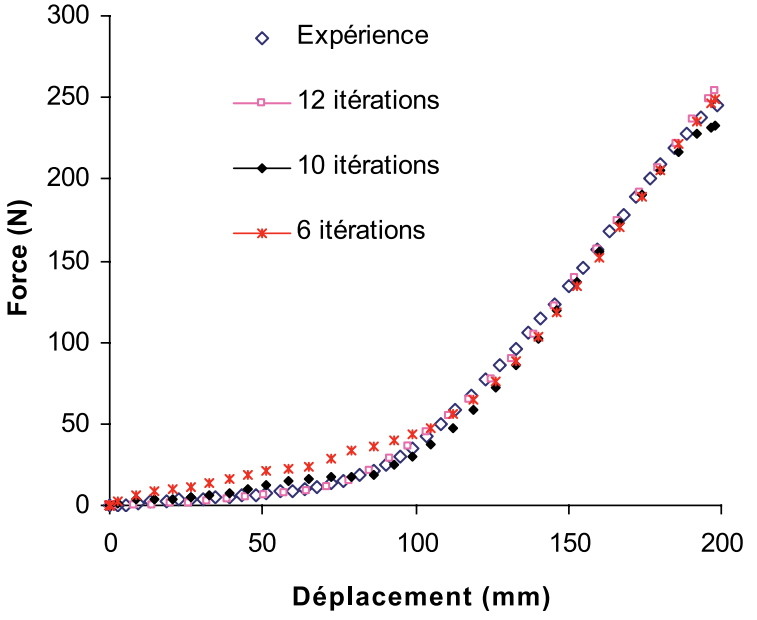

Fig. 6. Réponse globale : méthode de Levenberg-Marquardt.

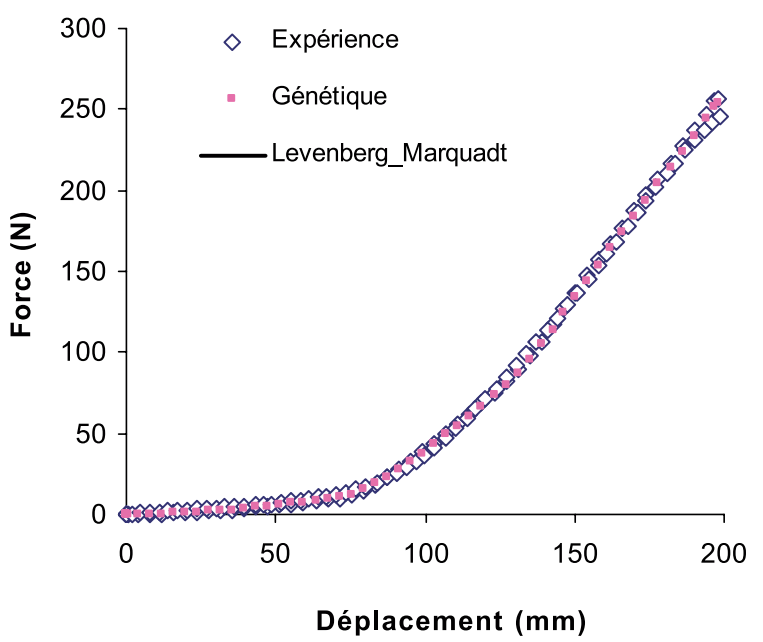

Fig. 7. Réponse globale : génétique - Levenberg-Marquardt. 


\section{Procédure de remaillage par raffinement}

Lors de la simulation de mise en forme, les éléments sont souvent soumis à de fortes sollicitations ce qui cause leurs distorsions et la divergence du calcul numérique. Pour cela, nous proposons une procédure de remaillage par raffinement couplée avec ABAQUS afin d'adapter le maillage à la forme géométrique souhaitée en considérant un critère de courbure des éléments [10]. L'opération de remaillage consiste à subdiviser les éléments ayant dépassé une courbure limite fixée pour créer de nouveaux éléments avec une taille plus petite.

La configuration initiale est composée par un nombre de fibres suivant les deux directions chaîne et trame et chaque fibre est discrétisée par un certain nombre d'éléments de barres. La procédure de remaillage par raffinement se base sur les deux hypothèses suivantes :

- Le nombre de fibres reste le même au cours du remaillage alors que le nombre d'éléments de barre constituant la fibre peut varier.

- Les éléments de membrane peuvent être subdivisés pour générer d'autres éléments de membrane de forme triangulaire et quadrangulaire.

La figure 9 présente la procédure de raffinement de maillage. Dans tous les cas les éléments de barres sont créés sur les arêtes de l'élément dans la configuration initiale [11]. L'algorithme de remaillage présenté sur la figure 8 passe par différentes étapes :

1. La première étape représente l'incrément de chargement réalisé sur la configuration initiale.

2. La deuxième étape intervient à la fin du chargement et elle consiste à calculer les valeurs des champs de déplacements et de contraintes dans les éléments.

3. La troisième étape consiste à tester le critère de remaillage si ce critère est vérifié la structure sera remaillée et de nouveaux éléments seront créés. La quatrième étape consiste à interpoler les champs calculés sur les nouveaux éléments en cas de remaillage. Si le nombre d'éléments reste le même il suffit juste de transférer les champs pour en tenir compte dans les conditions initiales de la nouvelle configuration.

Par illustration pour le premier niveau de raffinement : Dans $C_{0}$ les connectivités [1-2, 4-3] sont de type barre (trame), [1-4, 2-3] sont de type barre (chaîne) et [1-2-34] de type membrane (élasthanne). Dans $C_{\mathrm{t}}$ les connectivités $[1-2,3-5,4-5]$ sont de type barre (trame), [1-4, $2-3]$ sont de type barre (chaîne) et $[1-4-5,1-2-5,2-3-5]$ de type membrane (élasthanne).

\section{Exemple d'application : mise en forme par emboutissage}

Plusieurs problématiques peuvent être rencontrées lors de la mise en forme, parmi elles on peut citer la rupture des fibres au cours de l'emboutissage, l'apparition de plis et parfois l'impossibilité de réaliser une forme

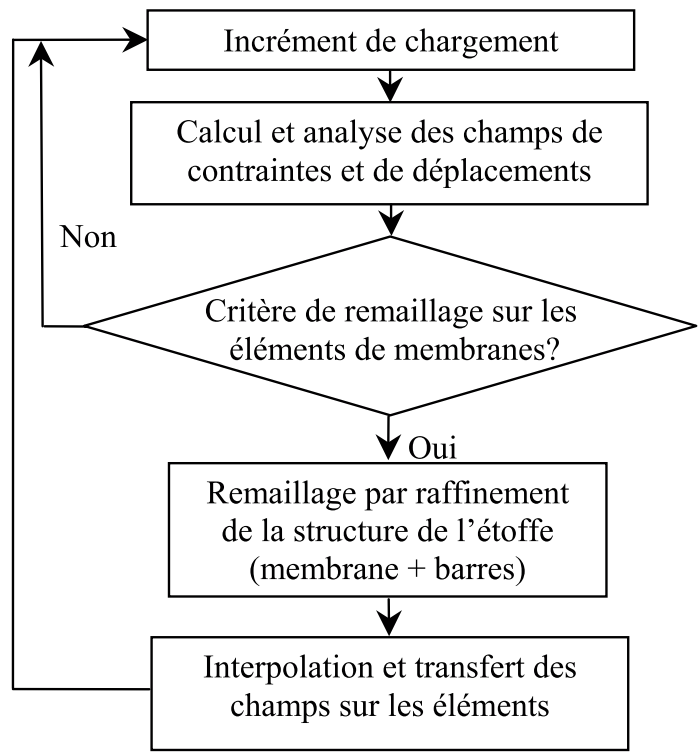

Fig. 8. Procédure de remaillage adaptatif des étoffes par raffinement.

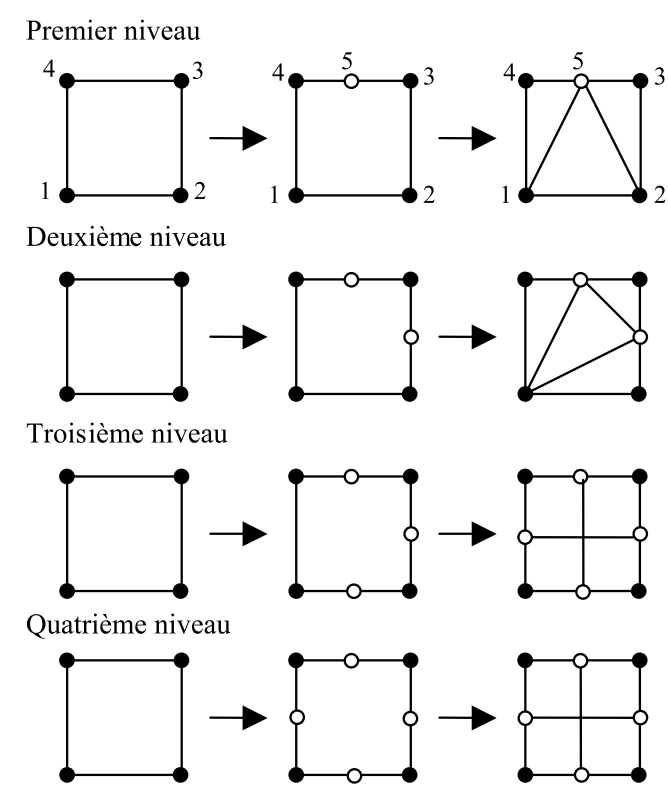

Fig. 9. Niveau de raffinement des étoffes.

géométrique. Pour cela et pour cerner ces problématiques, la simulation représente un bon outil pour prédire ces défauts et pour tester la faisabilité de la mise en forme. Deux exemples de simulation de mise en forme de structures souples sont présentés. Dans les deux cas on utilise une discrétisation géométrique avec les éléments finis décrits ci-dessus. Deux approches sont étudiées, la première traite le cas d'un comportement identique pour les deux directions de tissage (chaîne et trame) de la structure et la deuxième traite la cas de deux comportements différents. Les deux exemples étudiés représentent un cas de mise en forme isotherme avec un poinçon de forme conique. Le but de ces deux simulations est de prédire la limite de déformation en 3D de l'étoffe lors 


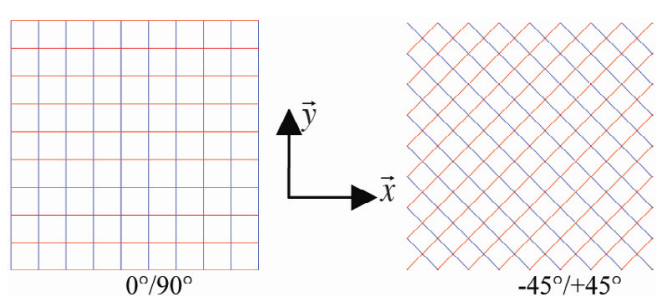

Fig. 10. Structures initiales de l'étoffe.

de l'emboutissage. Elles permettent de même de montrer les défaillances qui peuvent apparaître, telles les plis qui sont caractérisés par les grandes distorsions ou grandes déformations de compression.

\section{1 Étoffes avec comportement équilibré}

Les paramètres des deux matériaux utilisés sont déterminés par identification sur un essai de traction uni-axiale (voir Tab. 1). Les comportements des chaînes et des trames sont identiques et les propriétés sont intégrées sur ABAQUS/Explicite via la procédure matériau (VUMAT). Les deux exemples de simulation consistent à réaliser un essai d'emboutissage par poinçon conique sur une structure tissée composée de fibres chaîne et trame initialement orthogonales. La seule différence se situe au niveau de la position des directions des fibres par rapport aux directions principales des outils. Pour le premier, la direction des chaînes est à $0^{\circ}$ par l'axe $\mathrm{O} x$ alors que dans le deuxième exemple elle se situe à $45^{\circ}$. La forme de la structure $\left(0^{\circ} / 90^{\circ}\right)$ et $\left(-45^{\circ} / 45^{\circ}\right)$ à emboutir est présentée sur la figure 10 .

Le dispositif d'emboutissage est formé d'une matrice fixe, d'un serre-flan qui permet de maintenir la structure sans application d'effort extérieur et d'un poinçon de forme conique (voir Fig. 11). Les deux essais sont réalisés dans un premier temps sans la procédure de remaillage. Ils ont été de même réalisés expérimentalement [12].

La simulation du premier exemple a permis d'avoir une forme géométrique similaire à celle obtenue expérimentalement (Fig. 12). La disposition des fibres $\left(0^{\circ} / 90^{\circ}\right)$ favorise un retrait suivant la médiane de la structure. Ce retrait est symétrique par rapport au centre de l'étoffe. Pour l'essai à $45^{\circ}$, on enregistre un retrait au niveau des sommets puisque les fibres sont positionnées suivant les diagonales (Fig. 13).

Lors des deux simulations on enregistre des distorsions angulaires suivant la diagonale pour le premier exemple et suivant la médiane pour le deuxième. Les valeurs des distorsions sont relevées lors des essais expérimentaux et elles sont comparées aux valeurs numériques.

Ces valeurs sont mesurées sur la diagonale pour l'essai à $0^{\circ}$ et sur la médiane pour l'essai à $45^{\circ}$. Sur la figure 14, on peut remarquer que la distorsion atteint une valeur maximale à mi-distance entre le centre de la structure et ses sommets. Elle reste assez faible au niveau du centre et des sommets puisque la structure ne subit pas de grande déformation à ce niveau. On note que les diagonales de la préforme sont des lignes de fortes distorsions

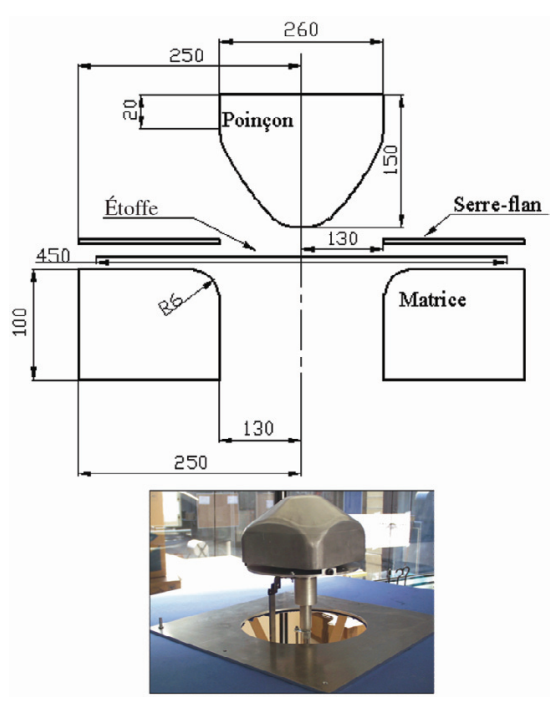

Fig. 11. Géométrie des outils de mise en forme.

angulaires $\left(43^{\circ}\right)$ dans le cas d'un emboutissage à $0^{\circ} / 90^{\circ}$ et que les médianes sont des lignes de fortes distorsions angulaires $\left(38^{\circ}\right)$ dans le cas de l'emboutissage $\left(-45^{\circ} / 45^{\circ}\right)$. $\mathrm{Au}$ niveau des courbes (Fig. 15), on obtient une bonne corrélation entre les valeurs expérimentales et les valeurs numériques.

Ces deux simulations sont réalisées dans un deuxième temps avec la procédure de remaillage adaptatif. On remarque sur la figure 16 que le retrait est moins accentué au niveau de la diagonale. Quatre zones de fortes distorsions angulaires sont remaillées. Le raffinement de la taille de l'élément permet d'adapter la taille des éléments à la géométrie souhaitée. Au niveau de l'exemple à $45^{\circ}$, on remarque de même que le raffinement des éléments se fait au niveau de la médiane dans les zones à forte déformation angulaire.

On trouve quasiment des valeurs identiques pour le retrait des sommets puisque les fibres n'ont pas été remaillées suivant la diagonale.

Dans les deux exemples étudiés, l'approche de discrétisation par les éléments de barre et de membrane a permis d'avoir des résultats numériques cohérents par rapport aux résultats expérimentaux. D'après les deux essais, on peut remarquer que l'utilisation de la procédure de remaillage est liée à la rigidité de la structure. Elle est mieux adaptée pour les structures assez souples.

\section{2 Étoffes avec comportement non équilibré}

Les deux exemples de simulation ci-dessous sont réalisés avec des comportements différents pour les deux directions des fibres (Fig. 17). Dans cet exemple nous travaillons avec des fibres chaînes plus rigides que les trames. La géométrie obtenue est similaire à celle du comportement équilibré. Malgré cette différence de comportement, on n'enregistre qu'un léger décalage entre les contours (Fig. 18). En effet, le serre-flan applique un effort très faible sur l'étoffe ce qui favorise son glissement sur la 

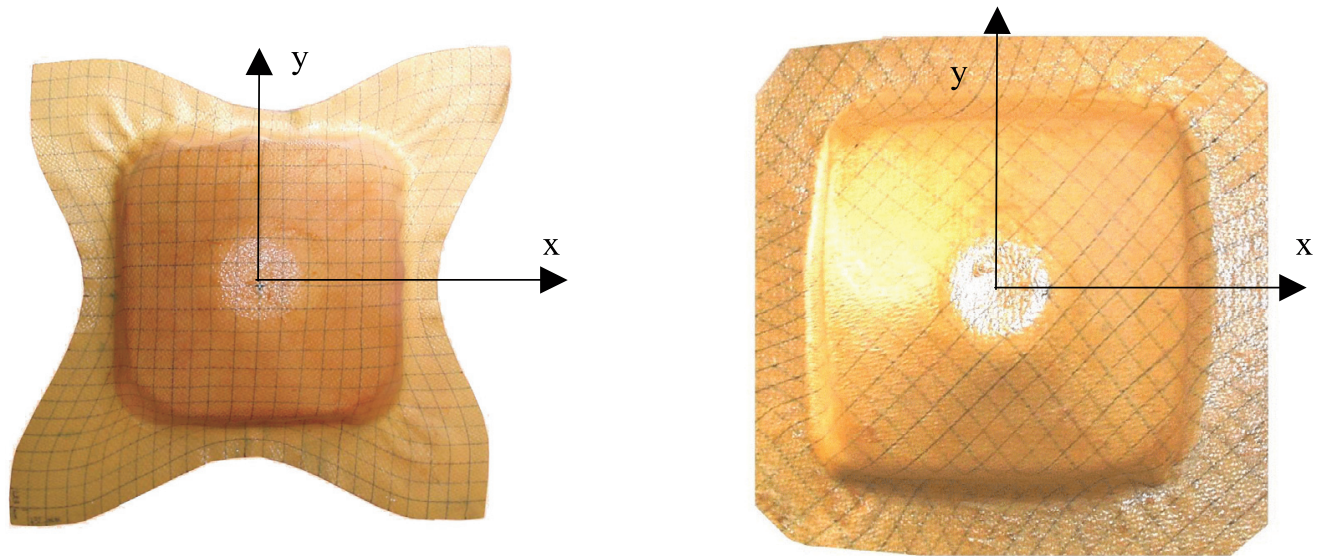

Fig. 12. Mise en forme : essai expérimental : (a) $0^{\circ} / 90^{\circ}$ et (b) $-45^{\circ} / 45^{\circ}$.
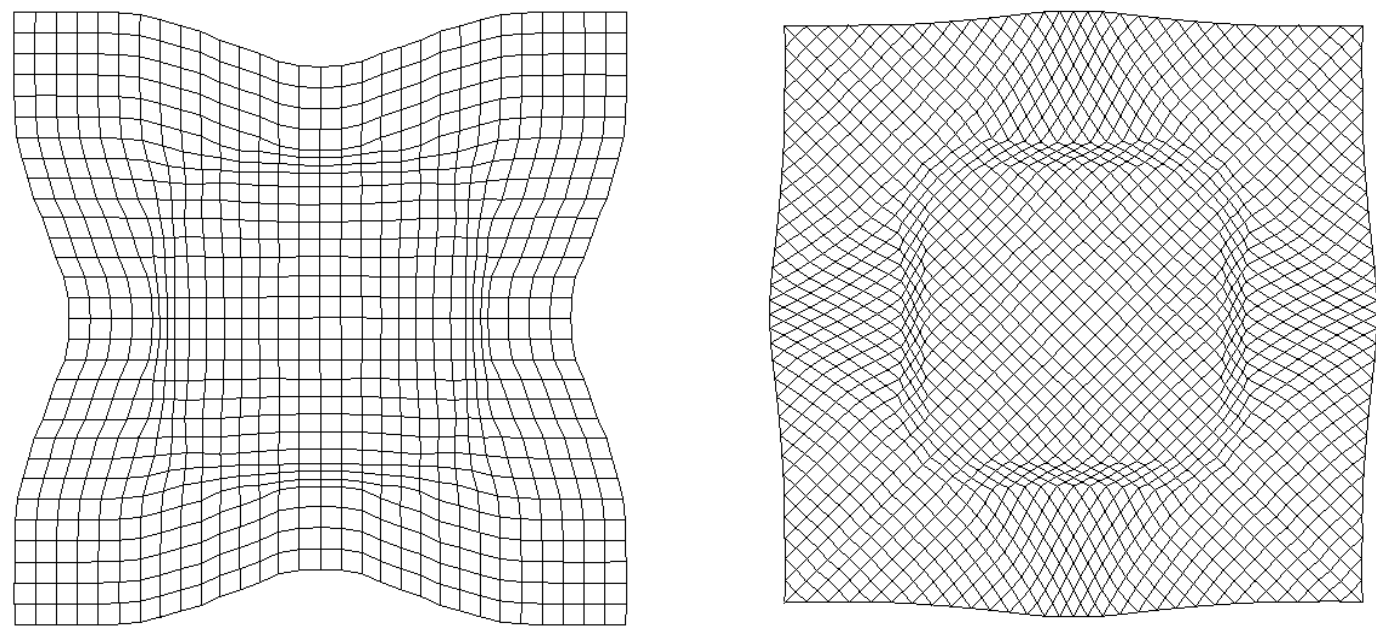

Fig. 13. Mise en forme : maillage déformé (a) $0^{\circ} / 90^{\circ}$ et (b) $-45^{\circ} / 45^{\circ}$.

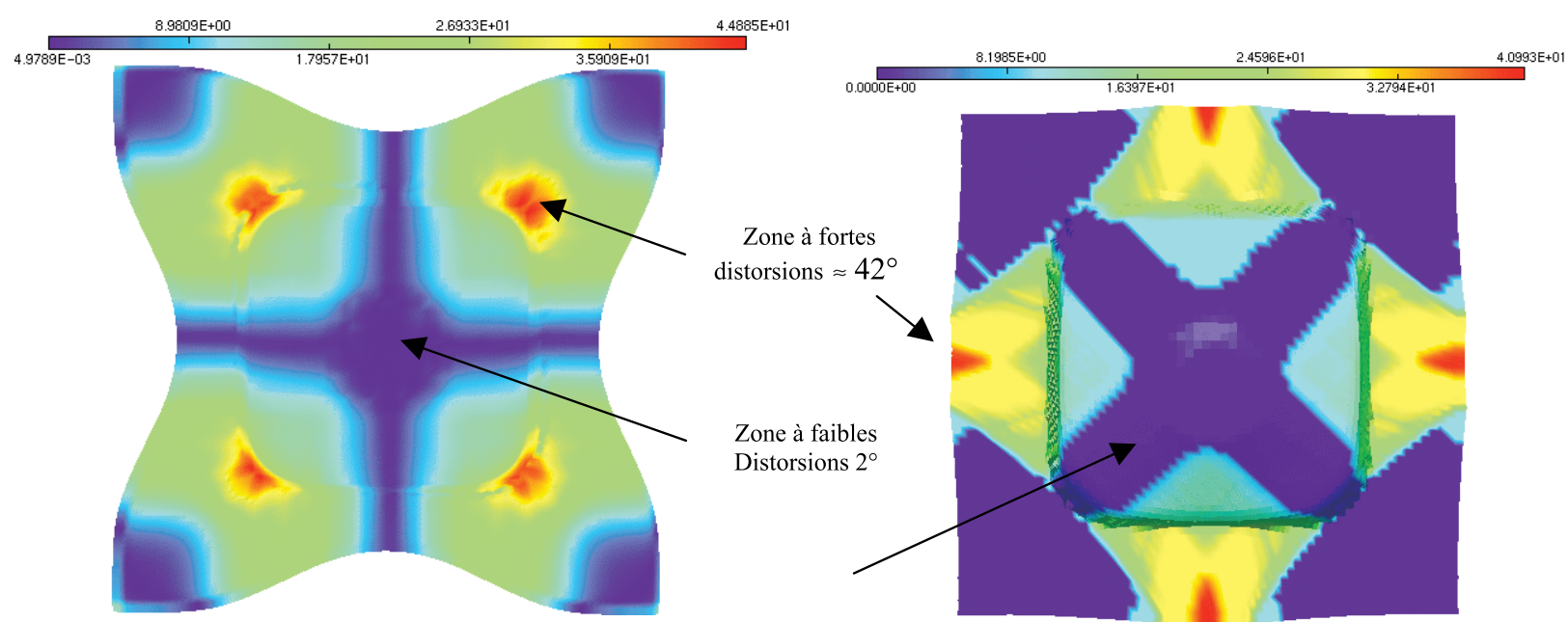

Fig. 14. Distorsions angulaires entre les fibres chaîne et trame. 

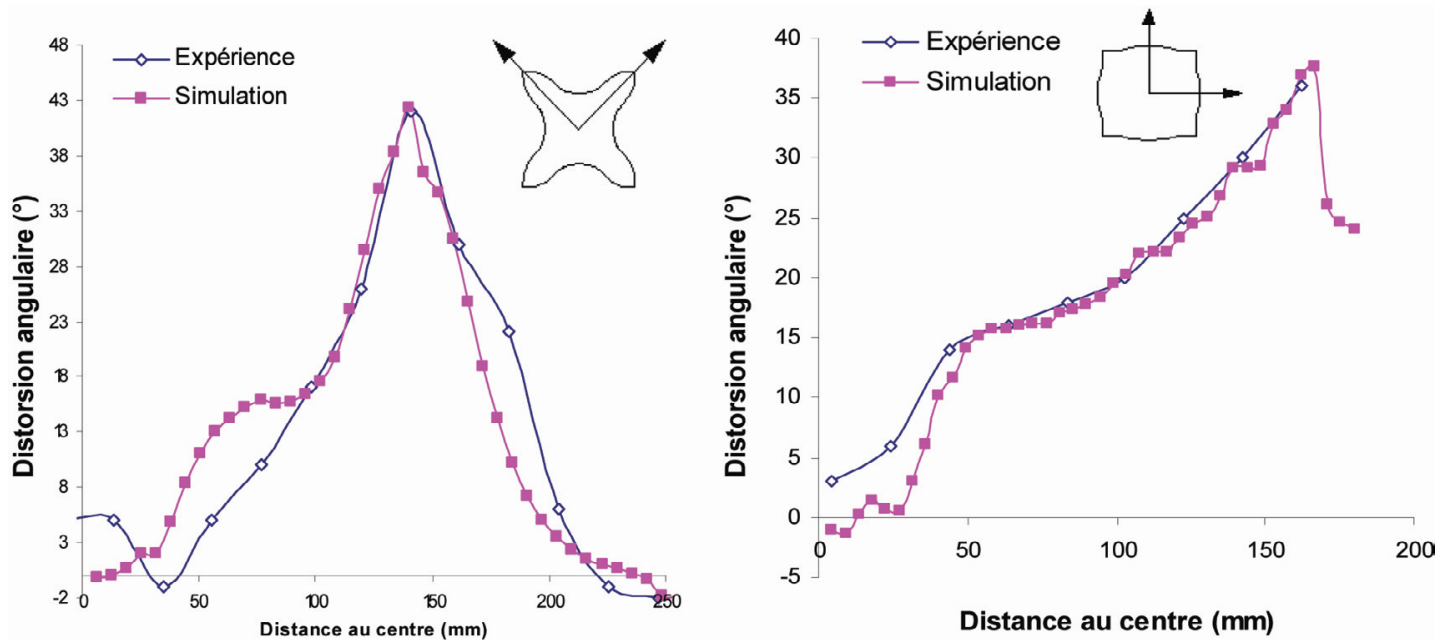

Fig. 15. Variation de la distorsion angulaire le long de la diagonale et la médiane de l'étoffe.
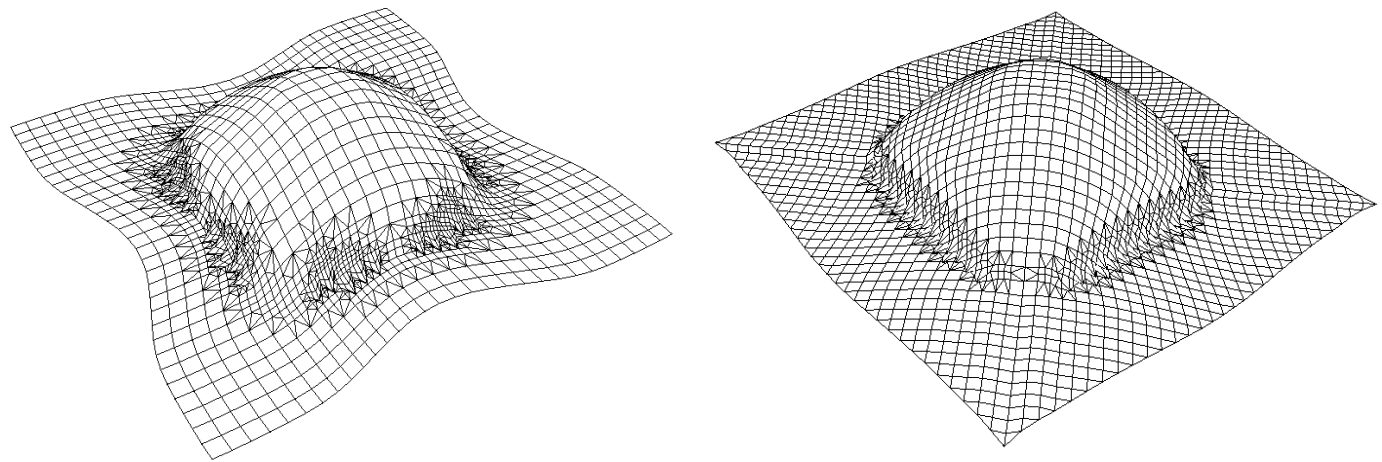

Fig. 16. Emboutissage avec remaillage adaptatif (a) $0^{\circ} / 90^{\circ}$ et (b) $-45^{\circ} / 45^{\circ}$.

matrice. Pour mieux voir l'influence du comportement des fibres sur le contour final de la préforme, il sera nécessaire d'appliquer une pression importante sous le serre-flan.

Pour l'essai à $0^{\circ}$, on enregistre des déplacements différents au niveau de la médiane. Le retrait est légèrement plus grand dans la direction des chaînes. Par rapport à l'essai à $45^{\circ}$, on ne remarque pas une grande différence au niveau des débordements sur les quatre arêtes. De même pour les distorsions angulaires, on trouve des valeurs comparables à celles calculées lors de l'emboutissage avec comportement équilibré.

Le modèle considéré dans cette partie est de la forme : $\sigma_{\mathrm{L}}=E_{0}+E_{1} \cdot \varepsilon_{\mathrm{L}}+E_{2} \cdot \varepsilon_{\mathrm{L}}^{2}+E_{3} \cdot \varepsilon_{\mathrm{L}}^{3}+E_{4} \cdot \varepsilon_{\mathrm{L}}^{4}+E_{5} \cdot \varepsilon_{\mathrm{L}}^{5}$.

Les paramètres des fibres de chaîne et trame sont identifiés par un essai de traction uni-axiale. Les valeurs sont résumées sur le tableau 2.

\section{Conclusion}

La procédure d'identification par méthode inverse d'un modèle de comportement macromécanique permet de déterminer les paramètres physiques des structures souples. L'identification est réalisée sur des essais de traction uni-axiale. Cette méthode est testée avec deux algorithmes d'optimisation, le premier c'est l'algorithme

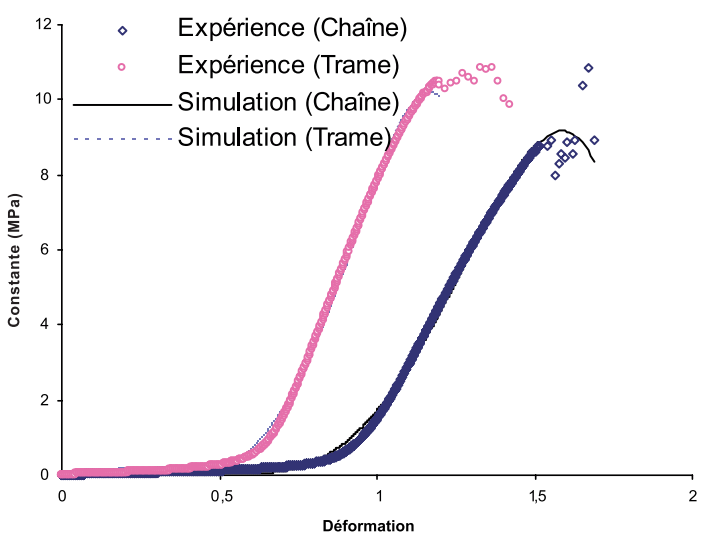

Fig. 17. Comportement non équilibré des fibres chaîne et trame.

Tableau 2. Valeurs des paramètres du modèle pour chaînes et trames.

\begin{tabular}{lll}
\hline Coefficients & Sens Chaînes & Sens Trame \\
\hline$E_{0}$ & $-0,0385$ & 0,0041 \\
$E_{1}$ & 0,3087 & $-1,0455$ \\
$E_{2}$ & 13,0354 & 13,8683 \\
$E_{3}$ & $-73,7609$ & $-41,5456$ \\
$E_{4}$ & 124,3321 & 44,1245 \\
$E_{5}$ & $-56,0357$ & $-13,7026$ \\
\hline
\end{tabular}



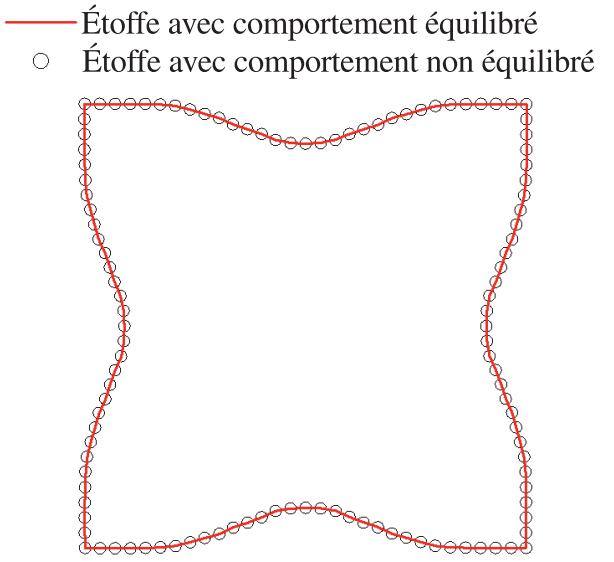

Étoffe avec comportement équilibré

- Étoffe avec comportement non équilibré

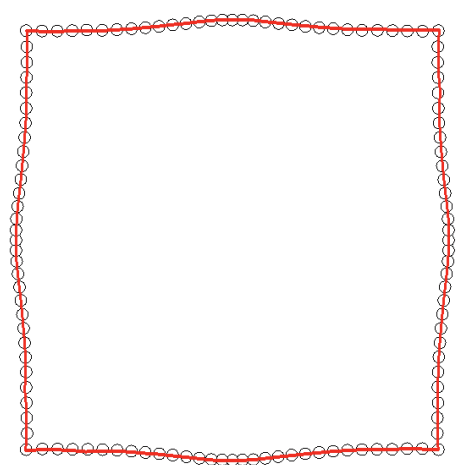

Fig. 18. Contour des étoffes $0^{\circ} / 90^{\circ}$ et $-45^{\circ} /+45^{\circ}$ avec comportement équilibré et non équilibré.

génétique qui a l'avantage d'éviter les minimums locaux et le deuxième est celui de Levenberg-Marquardt qui a l'avantage de la rapidité de convergence. La simulation numérique de la mise en forme des étoffes avec remaillage adaptatif présente un outil avantageux pour la prédiction de la faisabilité de l'opération de mise en forme. Deux exemples d'emboutissage à $0^{\circ}$ et $45^{\circ}$ sont réalisés sur une structure taffetas. Ces deux essais ont permis d'avoir une idée sur la capacité des étoffes aux grandes déformations en $3 \mathrm{D}$. Au niveau des résultats numériques on obtient une cohérence par rapport aux essais expérimentaux. Ces résultats peuvent être améliorés avec l'intégration d'un modèle de comportement tenant compte de l'effet d'interaction entre les fibres chaînes et les fibres trames.

\section{Références}

[1] O. Nocent, Animation dynamique de corps déformables continus : application à la simulation de textiles tricotés, Thèse de doctorat, université de Reims ChampagneArdenne, 2001

[2] T.-C. Lim, S. Ramakrishna, Modelling of composite sheet forming: a review, Composites Part A: Applied science and manufacturing 33 (2002) 515-537

[3] M.K. Warby, J.R. Whiteman, W.-G. Jiang, P. Warwick, T. Wright, Finite element simulation of thermoforming processes for polymer sheets, Mathematics and computers in simulation 61 (2003) 209-218
[4] X. Peng, Jian Cao, A dual homogenization and finite element approach for material characterization of textile composites, Composites Part B: engineering 33 (2002) $45-56$

[5] B. Gommers, I. Verpoest, P. Van Houtte, Modelling the elastic properties of knitted fabric-reinforced Composites, Composites Science and Technology 56 (1996) 685-694

[6] Y. Luo, I. Verpoest, Biaxial tension and ultimate deformation of knitted fabric reinforcements, Composites Part A: applied science and manufacturing 33 (2002) 197-203

[7] L. Giraud-Moreau, P. Lafon, Comparison of evolutionary algorithms for mechanical design components, Engineering optimisation 34 (2002) 307-322

[8] I. Ben Naceur, A. Cherouat, H. Borouchaki, J.M. Bachmann, Caractérisation et modélisation de l'aptitude à la déformation des structures souples, Revue des composites et des matériaux avancés 13 (2003) 231-240

[9] ZEBULON, Z-mat Version 8.8, Northwest Numerics Inc

[10] H. Borouchaki, A. Cherouat, P. Laug, K. Saanouni, Adaptative meshing for ductile fracture prediction in metal forming, Comptes Rendus Mécanique 330(10) (2002) $709-716$

[11] L. Giraud-Moreau, H. Borouchaki, A. Cherouat, Remaillage adaptatif pour la mise en forme des tôles minces, Comptes Rendus Mécanique 333(4) (2005) 371-378

[12] F. Penso, Adaptation d'un banc de simulation expérimentale, Réalisation d'essais de validation expérimentale : Simulation numérique d'essais de mise en forme, Université de Technologie de Troyes-ENSAM LM2S, 1999 